\title{
SOME RESULTS ON CHANNEL CAPACITY WHEN USING MULTIPLE ANTENNAS
}

\author{
Quentin H. Spencer \& A. Lee Swindlehurst \\ Department of Electrical \& Computer Engineering \\ Brigham Young University \\ Provo, Utah 84602 \\ spencerq@ee.byu.edu
}

\begin{abstract}
Recent research has demonstrated that the capacity of a multipath-rich channel (such as indoors) is dramatically increased when antenna arrays are used at both transmitter and receiver. This is achieved by using the multipath to create parallel sub-channels. This ability to achieve parallelism in the channel is influenced by how much information about the channel is known at the transmitter, the structure of the channel, and the structure of the arrays. This paper discusses the influence of the transmitter's knowledge of the channel on capacity, presents closed-form solutions for capacity when there are two paths in the channel, and then presents numerical results based on simulations. For the two-path case, the simulation data compare capacity based on differing assumptions about the transmitter, and compare different array structures. Simulation results are also presented for random channels generated using a statistical model for indoor multipath propagation.
\end{abstract}

\section{INTRODUCTION}

Recent research $[1,2,3]$ has shown that dramatic increases in channel capacity are possible in multipathrich environments when multiple antennas are used at both transmitter and receiver. The additional capacity is due to the fact that spatial diversity at both ends of the channel enables parallel transmission of data, something not possible when spatial diversity is present only at one end of the channel.

The results for capacity reported in the literature so far have subtle differences which are due to differing assumptions about the channel. In particular, what the transmitter knows about the channel has a great bearing on the transmission scheme, and ultimately the

This work was supported by the National Science Foundation under Wireless Initiative Grant CCR 99-79452. achievable capacity. The purpose of this paper is to compare, analytically and numerically, the capacity of the channel under varying assumptions about information available to the transmitter, differing array structures, and different models for the propagation in the channel. The next section provides more detail on these various assumptions. Section 3 presents a derivation for the channel capacity when the channel is low-rank and the transmitter has partial information about the channel. Section 4 presents a mathematical analysis of the singular values of $\mathbf{H}$ for a rank- 2 channel, and uses the result to derive a closed-form expression for capacity in terms of the gains and angles of the two paths for both the full information and partial information at the transmitter scenarios. Section 5 presents simulation results based on the two-path analysis, and Section 6 presents simulation results based on a realistic indoor propagation environment.

\section{BACKGROUND}

The capacity of a Multiple Input, Multiple Output (MIMO) channel is related to the singular values of the channel matrix $\mathbf{H}$. Assume there are $M_{T}$ transmitters and $M_{R}$ receivers, and that the channel is narrowband, where $h_{i j}$ represents the complex gain from the $i$ th transmitter to the $j$ th receiver. If $\mathbf{s}$ is the $M_{T} \mathrm{X}$ 1 vector of transmitted signal signals and $\boldsymbol{\eta}$ is additive noise, the received signal at the transmitter can be represented as

$$
\begin{gathered}
{\left[\begin{array}{c}
x_{1} \\
x_{2} \\
\vdots \\
x_{M_{R}}
\end{array}\right]=\left[\begin{array}{ccc}
h_{11} & \ldots & h_{1 M_{T}} \\
h_{21} & \ldots & h_{2 M_{T}} \\
\vdots & \ddots & \vdots \\
h_{M_{R} 1} & \ldots & h_{M_{R} M_{T}}
\end{array}\right]\left[\begin{array}{c}
s_{1} \\
s_{2} \\
\vdots \\
s_{M_{T}}
\end{array}\right]+\left[\begin{array}{c}
n_{1} \\
n_{2} \\
\vdots \\
n_{M_{R}}
\end{array}\right]} \\
\quad \mathbf{x}=\mathbf{H s}+\eta
\end{gathered}
$$


A common model used in rich scattering environments assumes that the elements of $\mathbf{H}$ are independent and identically distributed (complex) Gaussian random variables. For this assumption to be valid, the multipath scattering must be spatially uniform and the elements of the antenna array must be sufficiently separated.

In another model for the channel, the matrix $\mathbf{H}$ is factored into a sum over the individual multipath components of the channel, and their associated steering vectors (e.g., see [1]). For a narrow-band channel with $L$ paths, this sum is:

$$
\begin{aligned}
& \mathbf{H}=\sum_{l=1}^{L} \beta_{l}\left[\begin{array}{c}
a_{R, 1}\left(\theta_{R, l}\right) \\
\vdots \\
a_{R, M_{R}}\left(\theta_{R, l}\right)
\end{array}\right]\left[\begin{array}{lll}
a_{T, 1}\left(\theta_{T, l}\right) & \ldots & a_{T, M_{T}}\left(\theta_{T, l}\right)
\end{array}\right] \\
& =\left[\begin{array}{lll}
\boldsymbol{\alpha}_{R}\left(\theta_{R, 1}\right) & \ldots & \boldsymbol{\alpha}_{R}\left(\theta_{R, L}\right)
\end{array}\right]\left[\begin{array}{lll}
\beta_{1} & & 0 \\
& \ddots & \\
0 & & \beta_{L}
\end{array}\right]\left[\begin{array}{c}
\boldsymbol{\alpha}_{T}^{T}\left(\theta_{T, 1}\right) \\
\vdots \\
\boldsymbol{\alpha}_{T}^{T}\left(\theta_{T, L}\right)
\end{array}\right]
\end{aligned}
$$

where $\theta_{R, i}$ are the angles of arrival at the receiver, $\theta_{T, i}$ are the angles of departure at the transmitter, and $\beta_{l}$ is the complex gain of the $l^{\text {th }}$ path. The coefficient $a_{R, j}(\theta)$ is the complex gain of the $j^{\text {th }}$ receive array element to a signal arriving at angle $\theta$, and $a_{T, j}(\theta)$ is the gain of a signal launched in direction $\theta$ by the transmit array. These coefficients are collected into the steering vectors for the transmit and receive arrays for a given angle:

$$
\begin{aligned}
& \alpha_{T}(\theta)=\left[\begin{array}{lllll}
a_{T, 1}(\theta) & a_{T, 2}(\theta) & \ldots & a_{T, M_{T}}(\theta)
\end{array}\right]^{T} \\
& \alpha_{R}(\theta)=\left[\begin{array}{lllll}
a_{R, 1}(\theta) & a_{R, 2}(\theta) & \ldots & a_{R, M_{R}}(\theta)
\end{array}\right]^{T}
\end{aligned}
$$

Using this factorization of $\mathbf{H}$, the first channel we study in detail is the multipath channel with exactly two paths from transmitter to receiver. Channels in urban and indoor multipath environments will generally have considerably more than two paths, but this case is useful because its simplicity allows for some closed-form expressions for capacity in terms of the physical channel parameters, such as path angles and gains. It also illustrates the effects on capacity of the relative separation of angles for any two paths. Intuitively, two paths occuring very close to each other in angle will result in an $\mathbf{H}$ matrix of reduced rank, and thus a lower channel capacity. Numerical results based on the two-path analysis allow a comparison of capacity for different array structures, and illustrate the effects of the amount of channel information available at the transmitter on the capacity.

For simulating realistic channels encountered in indoor multipath applications, a statistical model was used [4]. This model groups multipath arrivals at the receiver into clusters in time and angle. Under the narrow-band assumption we use in this paper, the time delays of the paths are assumed to be small compared to the bandwidth of the system, and are therefore ignored, other than the fact that they are used in generating the amplitudes. The amplitudes are Rayleigh random variables whose means are decaying exponentials over each cluster. The clusters are distributed uniformly in angle, and the arrivals in each cluster have a Laplacian distribution with respect to the mean cluster angle. This model is discussed in more detail in [4].

\section{CAPACITY OF A LOW RANK CHANNEL}

In [1], capacity is derived for the case where the channel is deterministic and is known perfectly at the transmitter. In [2, 3], the channel is assumed to be random. For both cases, a corresponding optimal transmission scheme is chosen. Suppose that the matrix $\mathbf{H}$ is deterministic, and the transmitter knows only part of the information contained in $\mathbf{H}$. This could include knowledge of only the departure angles $\theta_{T, i}$, or it could additionally include knowledge of the path gains $\beta_{i}$. For either case, we assume $\mathbf{H}$ can be factored as $\mathbf{H}=\mathbf{A B}$, where $\mathbf{B}$ contains the information about the channel known at the transmitter. The matrix $\mathbf{B}$ is assumed to be of dimension $L \times M_{T}$, where $L \leq M_{T}$, and is full rank. Assuming in addition that $L \leq M_{R}$, it is shown in [1] that $L$ is the limiting factor in the capacity, and thus is the maximum achievable parallelism in the channel. In other words, the transmitted signal vector s of dimension $M_{T}$ can transmit only $L$ symbols at a time. Let $\tilde{\mathbf{s}}$ be the vector of $L$ symbols to be transmitted, and we will transmit some optimal linear combination of the symbols in $\tilde{\mathbf{s}}$ :

$$
\mathbf{s}=\mathbf{Y} \tilde{\mathbf{s}}
$$

where the matrix $\mathbf{Y}$ is a set of transmit vectors computed from our knowledge of B. With these assumptions, the received signal is $\mathbf{x}=\mathbf{A B Y} \tilde{\mathbf{s}}+\boldsymbol{\eta}$.

The capacity of this system is the base-2 logarithm of the determinant of the covariance matrix of the received signal vector $\mathbf{x}$, which takes the following form when the signal $\tilde{\mathbf{s}}$ is white and the noise has unit variance:

$$
C=\log _{2} \operatorname{det}\left(\mathbf{I}+\mathbf{A B Y} \mathbf{Y}^{*} \mathbf{B}^{*} \mathbf{A}^{*}\right)
$$

where $\mathbf{Y}$ is chosen to maximize the determinant subject to the power constraint. In cases of high signal to noise ratio, the second term in the determinant dominates, so to simplify we use the approximation proposed in 
[5], where the identity matrix is dropped. This gives

$$
\begin{aligned}
C & \approx \log _{2} \operatorname{det}\left(\mathbf{A B Y} \mathbf{Y}^{*} \mathbf{B}^{*} \mathbf{A}^{*}\right) \\
& =\log _{2}\left|\mathbf{A}^{*} \mathbf{A}\right|+\log _{2}\left|\mathbf{B Y} \mathbf{Y}^{*} \mathbf{B}^{*}\right|
\end{aligned}
$$

Now the capacity has been split into two terms, the first of which is independent of our knowledge of the channel, and the second of which must be optimized. A similar transmit vector optimization problem is addressed in $[6,7]$ in the context of space-time coding, where an optimal code must be selected at the transmitter to maximize the diversity gain. The problem in this case is to maximize the second term in (10) subject to the power constraint tr $\mathbf{Y}^{*} \mathbf{Y}=P$. Using a Lagrange multiplier $\lambda$, we take the derivative with respect to the matrix $\mathbf{Y}$ and set it to zero:

$$
\begin{gathered}
\frac{\partial}{\partial \mathbf{Y}} \log \left|\mathbf{B} \mathbf{Y} \mathbf{Y}^{*} \mathbf{B}^{*}\right|+\lambda\left(P-\operatorname{tr} \mathbf{Y}^{*} \mathbf{Y}\right)= \\
2 \mathbf{B}^{*}\left(\mathbf{B} \mathbf{Y} \mathbf{Y}^{*} \mathbf{B}^{*}\right)^{-1} \mathbf{B Y}-2 \lambda \mathbf{Y}=\mathbf{0} .
\end{gathered}
$$

This results in:

$$
\lambda \mathbf{Y}=\mathbf{B}^{*}\left(\mathbf{B Y} \mathbf{Y}^{*} \mathbf{B}^{*}\right)^{-1} \mathbf{B} \mathbf{Y} .
$$

Premultiplying both sides by $\mathbf{Y}^{*}$, taking a trace, and using the commutivity of matrices under the trace gives:

$$
\begin{aligned}
\lambda \operatorname{tr} \mathbf{Y}^{*} \mathbf{Y} & =\operatorname{tr} \mathbf{Y}^{*} \mathbf{B}^{*}\left(\mathbf{B} \mathbf{Y} \mathbf{Y}^{*} \mathbf{B}^{*}\right)^{-1} \mathbf{B Y} \\
& =\operatorname{tr} \mathbf{I}_{L}=L .
\end{aligned}
$$

Now, solving for $\lambda$ gives $\lambda=L / P$. Substituting this into the original expression gives an expression for $\mathbf{Y}$, which can be simplified one step further:

$$
\begin{aligned}
\mathbf{Y} & =\frac{P}{L} \mathbf{B}^{*}\left(\mathbf{B Y Y}^{*} \mathbf{B}^{*}\right)^{-1} \mathbf{B Y} \\
& =\frac{P}{L} \mathbf{B}^{*}\left(\mathbf{Y}^{*} \mathbf{B}^{*}\right)^{-1}
\end{aligned}
$$

Premultiplying $\mathbf{Y}$ by $\mathbf{Y}^{*}$ yields

$$
\mathbf{Y}^{*} \mathbf{Y}=\frac{P}{L} \mathbf{I}_{L},
$$

which shows that in order to maximize the determinant and achieve the capacity, the columns of $Y$ must be orthogonal.

Using the expression for $\mathbf{Y}$ and substituting it into $\mathbf{B Y Y} \mathbf{B}^{*}$ gives the second term of the capacity in (10):

$$
\begin{aligned}
& \mathbf{Y Y}^{*}=\frac{P}{L} \mathbf{B}^{*}\left(\mathbf{B} \mathbf{Y} \mathbf{Y}^{*} \mathbf{B}^{*}\right)^{-1} \mathbf{B} \mathbf{Y} \mathbf{Y}^{*} \\
& \mathbf{B Y} \mathbf{Y}^{*} \mathbf{B}^{*}=\frac{P}{L} \mathbf{B B}^{*}\left(\mathbf{B Y} \mathbf{Y}^{*} \mathbf{B}^{*}\right)^{-1} \mathbf{B} \mathbf{Y} \mathbf{Y}^{*} \mathbf{B}^{*} \\
&=\frac{P}{L} \mathbf{B B}^{*} \\
& \log _{2}\left|\mathbf{B} \mathbf{Y} \mathbf{Y}^{*} \mathbf{B}^{*}\right|=\log _{2}\left|\mathbf{B B}^{*}\right|+L \log _{2} \frac{P}{L}
\end{aligned}
$$

Substituting this back into the expression for capacity, it now simplifies as follows:

$$
\begin{aligned}
C & \approx \log _{2}\left|\mathbf{A} \mathbf{A}^{*}\right|+\log _{2}\left|\mathbf{B B}^{*}\right|+L \log _{2} \frac{P}{L} \\
& =\log _{2}\left|\mathbf{A B B} \mathbf{A B}^{*}\right|+L \log _{2} \frac{P}{L} \\
& =\log _{2}\left|\frac{P}{L} \mathbf{H H}^{*}\right|
\end{aligned}
$$

This is a function of the total transmitted power $P$. The mean power per transmit antenna will be $P / M_{T}$. Since the noise is assumed to have unit variance, the SNR per transmit antenna is also $P / M_{T}$. ¿From equations (18) and (22) it can be inferred that the optimal transmit vectors $\mathbf{Y}$ are a set of orthogonal vectors which span the column space of $\mathbf{B}^{*}$. The vectors are normalized to satisfy the power constraint.

\section{ANALYTICAL RESULTS FOR TWO PATHS}

\subsection{Singular values of $\mathbf{H}$}

When the channel has rank 2 , the singular values of $\mathbf{H}$, and therefore the capacity, has a closed form solution in terms of the steering vectors and path amplitudes. The channel matrix can be written as

$$
\mathbf{H}=\left[\begin{array}{ll}
\boldsymbol{\alpha}_{R}\left(\theta_{R, 1}\right) & \alpha_{R}\left(\theta_{R, 2}\right)
\end{array}\right]\left[\begin{array}{cc}
\beta_{1} & 0 \\
0 & \beta_{2}
\end{array}\right]\left[\begin{array}{l}
\boldsymbol{\alpha}_{T}^{T}\left(\theta_{T, 1}\right) \\
\boldsymbol{\alpha}_{T}^{T}\left(\theta_{T, 2}\right)
\end{array}\right]
$$

Grouping the gains with either set of steering vectors, we can write it as the product of two matrices:

$$
\mathbf{H}=\left[\begin{array}{ll}
\mathbf{a} & \mathbf{c}
\end{array}\right]\left[\begin{array}{c}
\mathbf{b}^{T} \\
\mathbf{d}^{T}
\end{array}\right]
$$

Using the Gram-Schmit procedure, the matrix on the left can be factored to simplify computation of the singular values:

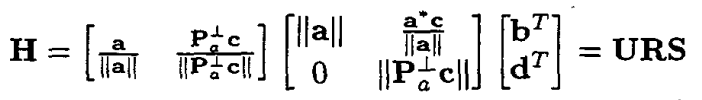

$$
\begin{aligned}
\mathbf{H H}^{*} & =\mathbf{U R S S}^{*} \mathbf{R}^{*} \mathbf{U}^{*} \\
\sigma^{2}(\mathbf{H}) & =\lambda\left(\mathbf{H H}^{*}\right)=\lambda\left(\mathbf{R S S}^{*} \mathbf{R}^{*}\right)
\end{aligned}
$$

So, by computing $\mathbf{R S S} \mathbf{R}^{*}$ and its eigenvalues we obtain the singular values of $\mathbf{H}$ needed to compute capacity. To simplify the final form of the singular values of $\mathbf{H}$, we normalize $\beta_{1}$ to 1 , and let $\beta_{2}=\beta e^{j \psi}$. An expression for the singular values in terms of the transmit and receive angles, the gain of the second arrival, and the number of transmit and receive antennas is included in the appendix. 


\subsection{Capacity for Full Channel Information at Transmitter}

When the transmitter knows the channel perfectly, the capacity as a function of the singular values, (from [1]), is

$$
C=\sum_{n=1}^{K} \log _{2}\left(1+\frac{\lambda_{S, n}\left|\lambda_{H, n}\right|^{2}}{\sigma^{2}}\right)
$$

where

$$
\lambda_{S, n}=\left(\xi-\frac{\sigma^{2}}{\left|\lambda_{H, n}\right|^{2}}\right)^{+} .
$$

In these equations, $\lambda_{H, n}$ is the $n^{\text {th }}$ singular value of $\mathbf{H}$ and $\lambda_{S, n}$ is the $n^{\text {th }}$ eigenvalue of $\mathbf{R}_{s s}$, the covariance matrix of the optimal transmitted data vector $\mathbf{s}$. The function $(\cdot)^{+}$is equal to its argument if it is positive and zero if it is negative. Since we have chosen to normalize $\beta_{1}=1$ for this discussion, we represent the noise power by the variable $\sigma^{2}$.

For the case of a rank- $2 \mathbf{H}$, a threshold can be calculated for which $\lambda_{S, 1}$ is some constant and $\lambda_{S, 2}$ is zero. The constant $\xi$ is set by the power constraint $P$. Since $P=\sum \lambda_{S, n}$, we can say that

$$
P=\left(\xi-\frac{\sigma^{2}}{\left|\lambda_{H, 1}\right|^{2}}\right)+\left(\xi-\frac{\sigma^{2}}{\left|\lambda_{H, 2}\right|^{2}}\right)^{+} .
$$

The second term in the sum is nonzero when

$$
\xi>\frac{\sigma^{2}}{\left|\lambda_{H, 2}\right|^{2}}
$$

Furthermore, from (33),

$$
\xi=\frac{1}{2}\left(P+\frac{\sigma^{2}}{\left|\lambda_{H, 1}\right|^{2}}+\frac{\sigma^{2}}{\left|\lambda_{H, 2}\right|^{2}}\right) .
$$

Combining these two equations results in a threshold necessary for achieving parallelism in the channel, that we write below in terms of the SNR per transmitted symbol, $\rho$ :

$$
\rho=\frac{P}{2 \sigma^{2}}>\frac{\left|\lambda_{H, 1}\right|^{2}-\left|\lambda_{H, 2}\right|^{2}}{2\left|\lambda_{H, 1}\right|^{2}\left|\lambda_{H, 2}\right|^{2}} .
$$

The threshold $\rho$ is a function of the array geometries and the five propagation variables: $\theta_{R, 1}, \theta_{R, 2}$, $\theta_{T, 1}$ and $\theta_{T, 2}$, and the relative amplitudes. To illustrate the effects of this threshold, Figure 1 plots $\rho$ for linear arrays of equal size at both ends of the channel. In this case, each path is assumed to have equal gain, $\theta_{R, 1}=\theta_{T, 1}=0$, and $\theta_{R, 2}=\theta_{T, 2}$, where the $\mathrm{x}$-axis in the plot is $\theta_{R, 2}$. As an illustration, suppose both arrays have four elements, and each path to the receiver

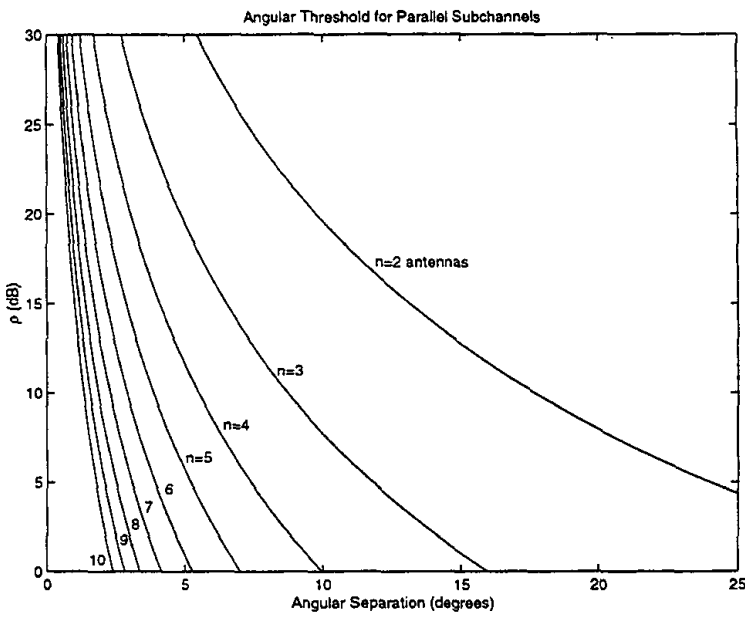

Figure 1: Angular threshold (as a function of $\rho$ ) necessary to achieve two parallel sub-channels for a linear array

has a signal to noise ratio of $15 \mathrm{~dB}$. This means that in order to achieve parallelism in the channel over two paths of equal amplitude, they must be separated by a minimum of $5^{\circ}$. With a linear array, this number will increase as the angles get further away from $0^{\circ}$. For any array structure, this number will also increase the more the amplitudes of the two paths differ.

Continuing the derivation of capacity, we now solve for $\xi$ :

$$
\xi= \begin{cases}\frac{1}{2}\left(P+\frac{\sigma^{2}}{\left|\lambda_{H, 1}\right|^{2}}+\frac{\sigma^{2}}{\left|\lambda_{H, 2}\right|^{2}}\right), & \rho>\frac{\left|\lambda_{H, 1}\right|^{2}-\left|\lambda_{H, 2}\right|^{2}}{2\left|\lambda_{H, 1}\right|^{2}\left|\lambda_{H, 2}\right|^{2}} \\ P+\frac{\sigma^{2}}{\left|\lambda_{H, 1}\right|^{2}}, & \rho \leq \frac{\left|\lambda_{H, 1}\right|^{2}-\left|\lambda_{H, 2}\right|^{2}}{2\left|\lambda_{H, 1}\right|^{2}\left|\lambda_{H, 2}\right|^{2}}\end{cases}
$$

Now it is possible to solve for $\lambda_{S, 1}$ and $\lambda_{S, 2}$. When $\rho$ is less than the threshold, there is only one possible channel, so $\lambda_{S, 1}=P$ and $\lambda_{S, 2}=0$. Otherwise, the solution is:

$$
\begin{aligned}
& \lambda_{S, 1}=\frac{P}{2}+\sigma^{2} \frac{\left|\lambda_{H, 1}\right|^{2}-\left|\lambda_{H, 2}\right|^{2}}{2\left|\lambda_{H, 1}\right|^{2}\left|\lambda_{H, 2}\right|^{2}} \\
& \lambda_{S, 2}=\frac{P}{2}-\sigma^{2} \frac{\left|\lambda_{H, 1}\right|^{2}-\left|\lambda_{H, 2}\right|^{2}}{2\left|\lambda_{H, 1}\right|^{2}\left|\lambda_{H, 2}\right|^{2}}
\end{aligned}
$$

Substituting the solutions for $\lambda_{S, 1}, \lambda_{S, 2}, \lambda_{H, 1}$ and $\lambda_{H, 2}$ into (31) gives an expression for the capacity of the channel. There are two solutions, depending on whether the threshold is satisfied. We will refer to these as $C_{1}$ and $C_{2}$, respectively. Here we define SNR as the total power transmitted divided by the total noise power at the receivers, or $2 \rho$ in this case. It may in some cases make more sense to average the SNR over 
the number of transmit antennas, but in the results section which follows, comparisons of different array sizes are more relevant when the total transmit power is constrained to be the same for every case. The capacities $C_{1}$ and $C_{2}$ are:

$$
\begin{aligned}
C_{1}= & \log _{2}\left[1+\operatorname{SNR}\left|\lambda_{H, 1}\right|^{2}\right] \\
C_{2}= & \log _{2}\left[1+\left(\frac{\mathrm{SNR}}{2}+\frac{\left|\lambda_{H, 1}\right|^{2}-\left|\lambda_{H, 2}\right|^{2}}{2\left|\lambda_{H, 1}\right|^{2}\left|\lambda_{H, 2}\right|^{2}}\right)\left|\lambda_{H, 1}\right|^{2}\right] \\
& +\log _{2}\left[1+\left(\frac{\mathrm{SNR}}{2}-\frac{\left|\lambda_{H, 1}\right|^{2}-\left|\lambda_{H, 2}\right|^{2}}{2\left|\lambda_{H, 1}\right|^{2}\left|\lambda_{H, 2}\right|^{2}}\right)\left|\lambda_{H, 2}\right|^{2}\right]
\end{aligned}
$$

These results characterize the maximum achievable capacity in situations when the transmitter knows the channel perfectly and the channel has rank 2 .

\subsection{Capacity for Partial Channel Information at Transmitter}

The capacity expression in (25) when applied to the two path case is:

$$
\begin{aligned}
C & \approx \log _{2}\left|\frac{P}{2} \mathbf{H H}^{*}\right|=\log _{2}\left|\rho \mathbf{H H}^{*}\right| \\
& =\log _{2}\left(\frac{P}{2}\left|\lambda_{H, 1}\right|^{2}\left|\lambda_{H, 2}\right|^{2}\right) \\
& =\log _{2} \frac{\mathrm{SNR}}{2}+\log _{2}\left|\lambda_{H, 1}\right|^{2}\left|\lambda_{H, 2}\right|^{2}
\end{aligned}
$$

Where the SNR is defined in the same way as the previous section, and is in this case equal to $P$.

\section{RESULTS FOR THE TWO-PATH CHANNEL}

Most of the results presented in this section consist of probability densities. Channel capacity was previously characterized as a random variable in [2]. In that case, the channel matrix was assumed to be composed of i.i.d. Gaussian elements. In this case, we treat capacity as a random variable, but $\mathbf{H}$ is now a deterministic function of random parameters, specifically the angles of the multipath components, and their gains.

The results that follow all assume that the two paths are uniformly distributed over all angles, and are independent. The relative phase of the two paths is also uniformly distributed. For the cases presented in this section, the two paths both have equal amplitudes. Three array structures are compared: linear, circular, and $\mathrm{Y}$-shaped. A Y-shaped array consists of three linear sub-arrays positioned $120^{\circ}$ from each other. Unless otherwise noted, the linear arrays are spaced at $\lambda / 2$.
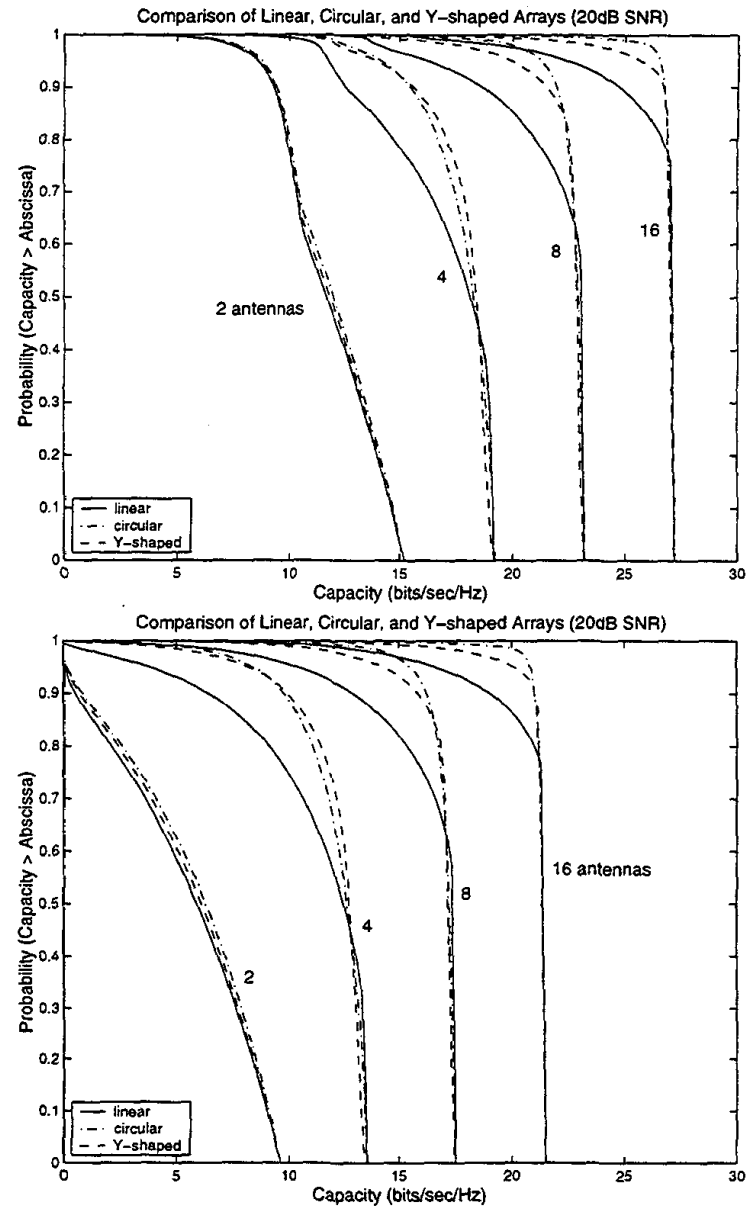

Figure 2: Capacity for different array sizes and structures when transmitter has complete channel knowledge (top), and partial channel knowledge(bottom).

The two other types of arrays are sized so that their overall diameter is equal to that of the linear array with the same number of elements. As a result, the three arrays compared here will have different element spacings, but the same overall aperture. In all cases, the number of antennas is assumed to be the same at transmitter and receiver (corresponding to a square $\mathbf{H}$ ).

In Figure 2, we compare capacity of the three array structures for $2,4,8$, and 16 antennas at $20 \mathrm{~dB}$ SNR for both of the assumptions about the transmitter discussed in this paper. The curves represent the complimentary cumulative density functions (CCDFs). The most noticeable feature is the difference in capacity between the two plots. Reduced information about the channel at the transmitter seems to produce curves with approximately the same shape, but a $20-30 \%$ re- 

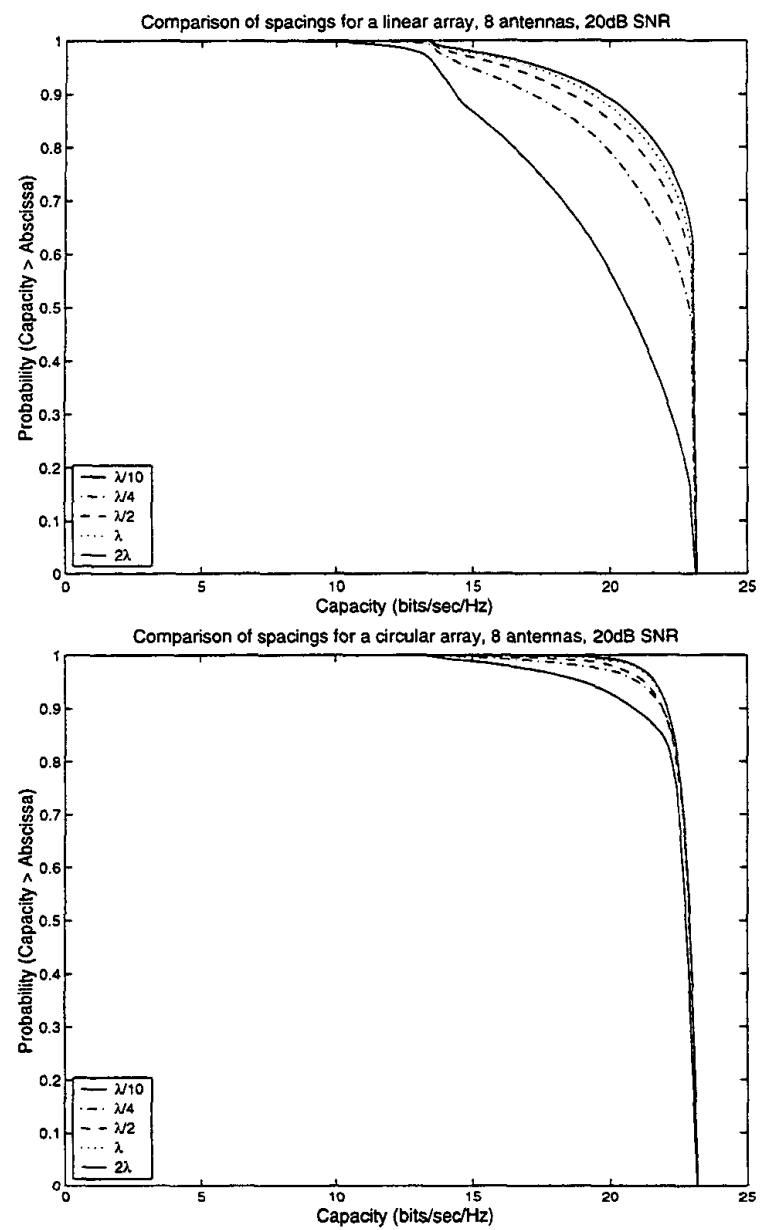

Figure 3: Effects of element spacings on capacity for linear (top) and circular arrays (bottom)

duction in available capacity. There is also a noticeable difference in performance between the different array structures. For low outage probabilities, the circular array gives slightly better performance than a $\mathrm{Y}$-shaped array, and significantly better performance than the linear array. The obvious deficiency in the performance of linear arrays is explained by two factors. First, linear arrays tend to have very poor angular resolution in "end-fire" configurations where the angle of arrival/departure is close to $\pm 90^{\circ}$ from broadside. The second problem is the fact that the steering vectors from a linear array are unique only over $180^{\circ}$. The Yshaped array was chosen as an alternative in this study because the three sub-arrays aligned at different angles is able to overcome this angular ambiguity. However, the circular array still gives the best performance.

Figure 3 shows the effects of changing the array ele-

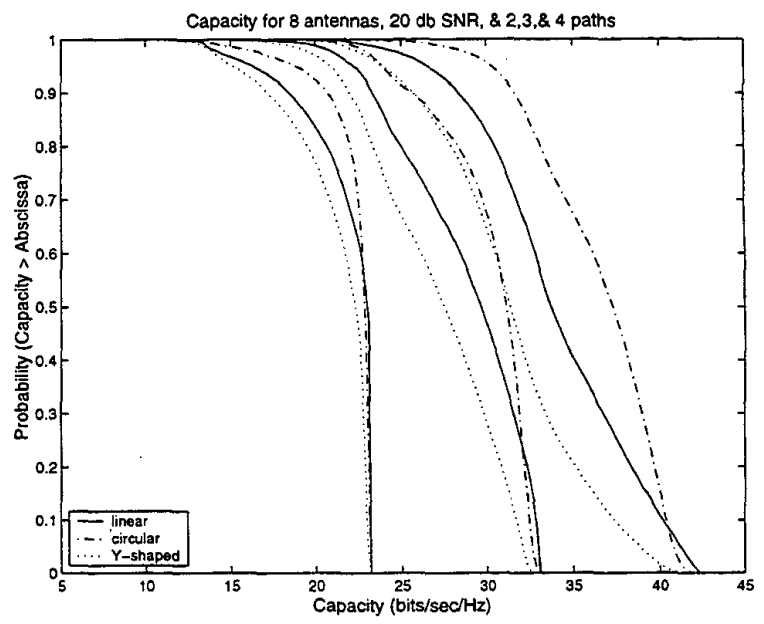

Figure 4: Comparison 2,3, and 4 paths at $20 \mathrm{~dB}$ SNR

ment spacing on capacity when the transmitter knows the channel completely. The spacing of an 8-element linear array was varied over the range from $\lambda / 10$ to $2 \lambda$, and compared with a circular array of the same aperture. As expected, as the spacing is decreased, the capacity begins to drop, and as the spacing increases, the increases in capacity become smaller as the capacity asymptotically approaches a maximum bound. The most interesting result is that when the spacing becomes very small, the gap between the capacities of the linear and circular arrays increases, and the circular array maintains a capacity very close that of its largest aperture.

To compare the results for two paths with capacity when there are more than two paths, Figure 4 shows the capacity for cases when there are two, three, and four paths. As the number of paths increases, the the capacity gradually increases, and the curves become more Gaussian. This is expected, since when is a very high number of paths, the matrix $\mathbf{H}$ will tend to be more Gaussian, and the elements will be more independent.

\section{RESULTS FOR THE INDOOR MULTI- PATH CHANNEL}

This section shows the results of simulations of a MIMO channel in an indoor multipath environment using a statistical model. The model parameters for selecting amplitudes and angles were taken from those measured for one of the buildings in [4].

Since the model generates angle of arrival only for the receiver, and no data are available to date on the transmitter angle of departure, we assumed that the transmitter angles share the same statistics and are 


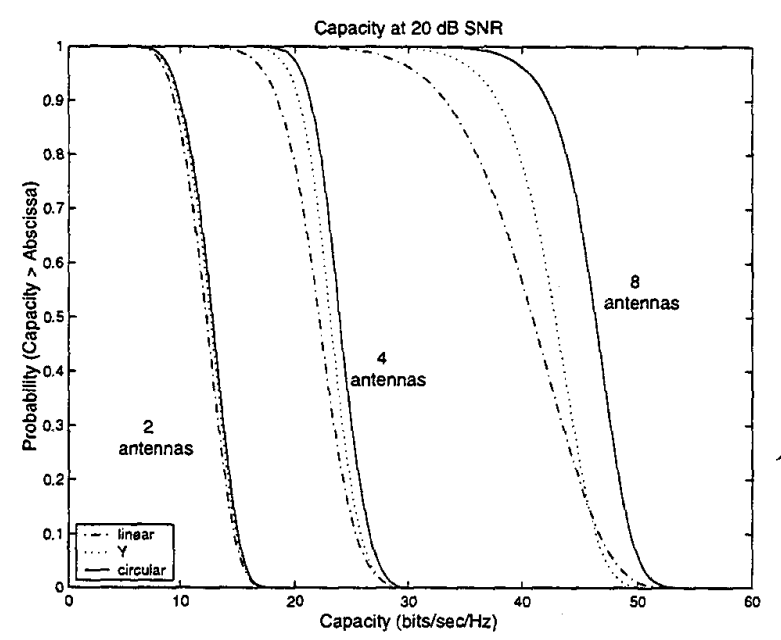

Figure 5: Comparison of capacity for array sizes of 2, 4 , and 8 elements for three different array structures

independent of those at the receiver. In the results shown here, the randomly chosen angles and gains were used to generate realizations of the matrix $\mathbf{H}$ for the array structures under test. A sufficiently large number of channel realizations gave enough data to generate probability densities of capacity.

Figure 5 shows some results of these simulations for array sizes of two, four, and eight elements. As before, the arrays are assumed to be the same size at both transmitter and receiver. Data are shown in this plot for linear, Y-shaped, and circular arrays. Once again, the circular array has the best performance, followed by the Y-shaped and linear arrays. The curves also seem to show that the capacity is approximately Gaussian. However, the curves do not perfectly fit a Gaussian distribution, as is shown in Figure 6. A Gaussian curve for the same mean and variance has been superimposed on the data, and it is clear that the general shape is Gaussian, but that there are asymmetries.

Figure 7 shows how the capacity per element changes as additional array elements are added. The array is a circular, and the number of its elements is varied from 2 to 30 . In [1], it is shown that if the channel is always full rank, the capacity per element approaches a constant as the array size is increased. In this simulation, however, the capacity per element continues to decrease as the arrays grow larger. This can by explained by the fact that the rank of the channel is large, but finite. The indoor multipath environment provides the necessary spatial diversity to achieve high capacity for reasonable sizes of arrays, but as the arrays become very large, the multipath environment eventually limits the rank of $\mathbf{H}$.

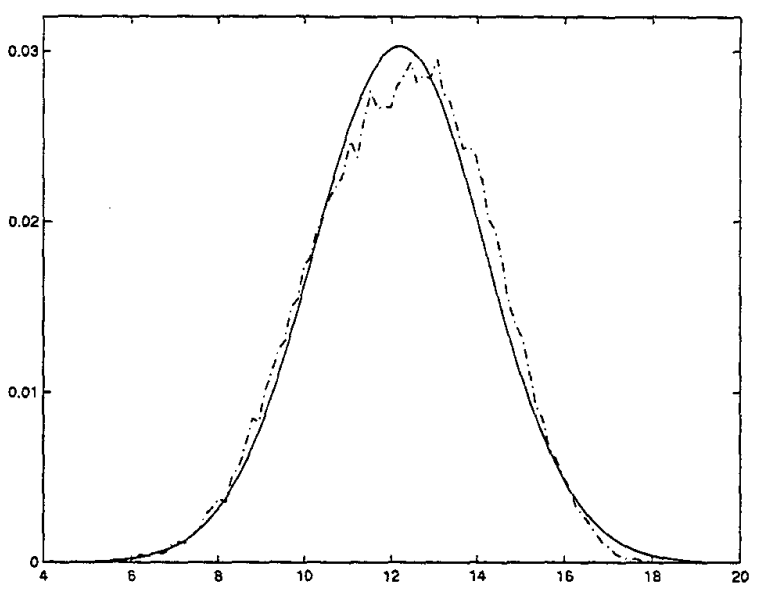

Figure 6: PDF of an indoor capacity simulation

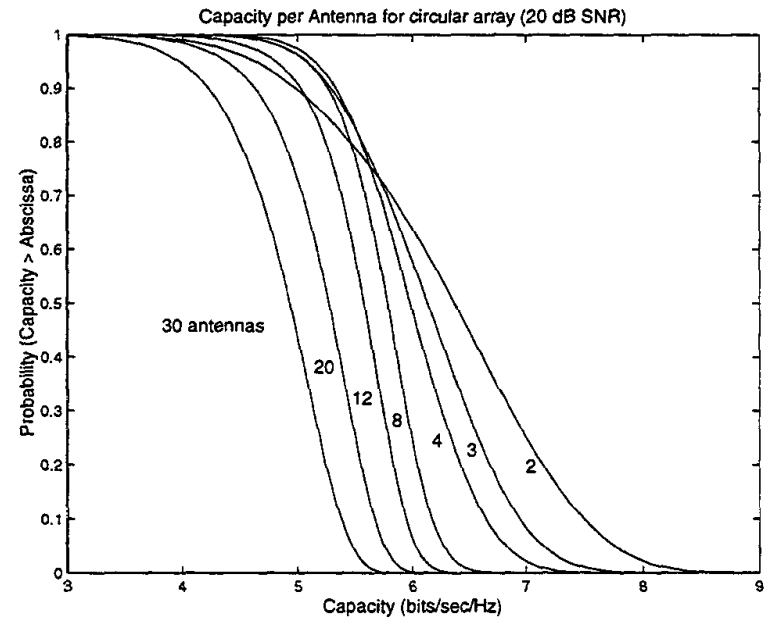

Figure 7: Capacity per array element as the array size increases for a circular array 


\section{CONCLUSION}

This paper has presented a solution for capacity of the MIMO channel when the channel is low rank and the transmitter has only partial information about the channel. For the cases of partial and full knowledge of the channel, a closed-form expression for capacity of a rank-2 channel was derived as a function of the steering vectors and path amplitudes. Using these analytical results, numerical simulations demonstrate a loss of $20-30 \%$ of available capacity when the transmitter has partial channel information. In addition, a comparison of three different array structures showed that when there are paths distributed over all angles, a circular structure gives the best performance. The circular array structure also retains its performance as the array spacing is reduced.

Simulations of an indoor multipath channel based on a statistical model demonstrate that the circular array continues to have the most desirable performance under more realistic conditions.

\section{APPENDIX A: SINGULAR VALUES OF A RANK-2 H}

The singular values of $\mathbf{H}$ in terms of the steering vectors as defined in Section 4 are:
[4] Q. H. Spencer, B. D. Jeffs, M. A. Jensen, and A. L. Swindlehurst. Modeling the statistical time and angle of arrival characteristics of an indoor multipath channel. IEEE Journal on Selected Areas In Communications, 18(3):347-360, March 2000.

[5] Dhananjay A. Gore, Rohit U. Nabar, and Arogyaswami Paulraj. Selecting an optimal set of transmit antennas for a low rank matrix channel. In Proceeings of ICASSP 2000. IEEE, 2000.

[6] Ardavan Maleki Tehrani, Rohit Negi, and John Cioffi. Space-time coding and transmission optimization for wireless channels. In Proceesings of Asilomar 1998. IEEE, 1998.

[7] Ardavan Maleki Tehrani, Rohit Negi, and John Cioffi. Space-time coding over a code division multiple acess system. In Proceedings of WCNC 1999. IEEE, 1999.

$$
\begin{aligned}
\sigma^{2}(\mathbf{H}) & =\frac{1}{2}\left(1+\beta^{2}\right) M_{T} M_{R}+\Re\left\{\beta e^{j \psi} \alpha_{R}\left(\theta_{R, 1}\right)^{*} \alpha_{R}\left(\theta_{R, 2}\right) \alpha_{T}\left(\theta_{T, 1}\right)^{*} \alpha_{T}\left(\theta_{T, 2}\right)\right\} \\
\pm & \sqrt{\begin{array}{r}
\left(\frac{1}{2}\left(1-\beta^{2}\right) M_{T} M_{R}+\Re\left\{\beta e^{j \psi} \alpha_{R}\left(\theta_{R, 1}\right)^{*} \alpha_{R}\left(\theta_{R, 2}\right) \alpha_{T}\left(\theta_{T, 1}\right)^{*} \alpha_{T}\left(\theta_{T, 2}\right)\right\}+\frac{\beta^{2} M_{R}}{M_{T}}\left|\alpha_{R}\left(\theta_{R, 1}\right)^{*} \alpha_{R}\left(\theta_{R, 2}\right)\right|^{2}\right)^{2} \\
+\left(\beta^{2}-\frac{\beta^{2}}{M_{T}^{2}}\left|\alpha_{R}\left(\theta_{R, 1}\right)^{*} \alpha_{R}\left(\theta_{R, 2}\right)\right|^{2}\right)\left|M_{T} \alpha_{T}\left(\theta_{T, 1}\right)^{*} \alpha_{T}\left(\theta_{T, 2}\right)+M_{R} \beta e^{j \psi} \alpha_{R}\left(\theta_{R, 2}\right)^{*} \alpha_{R}\left(\theta_{R, 1}\right)\right|^{2}
\end{array}}
\end{aligned}
$$

\section{REFERENCES}

[1] Gregory G. Raleigh and John M. Cioffi. Spatiotemporal coding for wireless communication. IEEE Transactions on Communications, 46(3):357-366, March 1998.

[2] G. J. Foschini and M. J. Gans. On limits of wireless personal communications in a fading environment when using multiple antennas. Wireless Personal Communications, 6(3):311-335, March 1998.

[3] I. Telatar. "Capacity of Multiple Antenna Gaussian Channels". ATET Technical Memorandum, June 1995. 